\title{
Oxygen Exchange Kineics of Thin Ftilms Studied by Optical Transmission Relaxation: Correlation with Surface Composition and Microstructure
}

\author{
S. R. Bishop, L. Zhao, T. Daio, N. Perry, and K. Sasaki \\ International Institute for Carbon Neutral Energy Research (WPI-I2CNER), Kyushu University Nishi- \\ ku Fukuoka, 819-0395
}

Thin film electroceramics are of great interest in gas sensors and solid oxide fuel cells (SOFCs), where the small dimensions lead to more rapid response times in gas detection and low ionic resistance in SOFC electrodes and membranes. Additionally, they provide a geometrically well-defined platform for systematic electrochemical investigations. In these proceedings, an in situ optical transmission relaxation (OTR) technique for studying the oxygen exchange rate of thin films, a key figure of merit of SOFC electrodes, is introduced. This technique makes use of changes in concentration of optically active defect centers with step changes in environmental variables such as oxygen partial pressure and temperature. The absence of potentially catalytic metal contacts, commonly used in electrical conductivity relaxation and through-plane resistance measurements, is a key advantage of this technique, thereby allowing study of the bare film. Interestingly, oxygen exchange kinetics, which often decay with time, have been found to more rapidly degrade with this technique. The origin of this decay in $(\mathrm{Pr}, \mathrm{Ce}) \mathrm{O}_{2}-\delta$ thin films is discussed, and new surface treatment techniques that reverse the degradation are presented, the study of which are facilitated by this contactless approach. The optical absorption technique is a promising in situ method for investigating the impact of surface chemistry on oxygen exchange.

In the first part of this project, we examined the surface chemistry of a thin film which displayed very poor electrode kinetics [1]. A SIMS depth profile is shown in figure 1. The sample in the figure had exhibited decreasing electrode kinetics during the course of several days of OTR measurements at 300 $400^{\circ} \mathrm{C}$. Later, the sample was heated to $600^{\circ} \mathrm{C}$ and electrode kinetics were significantly lower than that previously found by more conventional electrical measurements. The SIMS depth profile clearly shows a large amount of $\mathrm{K}, \mathrm{Na}, \mathrm{Si}$, and $\mathrm{Ca}$ impurities at the surface of the film, extending approximately $60 \mathrm{~nm}$ into the film. In comparison, a newly deposited film did not show any impurity accumulation at the surface.

The impurity build-up at the surface is believed, at present, to be the primary source for previously measured degradation in oxygen surface exchange coefficient with time for this film. It is well known that Si (present in figure 1) significantly increases oxide ion grain boundary resistance and as well as blocking oxygen exchange at the surface, whilst surface $\mathrm{CaO}$ coverage has been shown to have a deleterious effect on surface oxygen exchange in yttria stabilized zirconia (a similar fluorite oxide), and has previously been observed on ceria based oxide surfaces.

In the second part of this project we developed a new method to enhance the electrode kinetics by using a surface treatment of La oxide [2]. Previous researchers have found that La oxide deposited on other state-ofthe-art SOFC electrodes improves performance, though the origin of the improvement is not well understood. In this case, we used pulsed laser deposition (PLD) to deposit La oxide on the surface of a PCO film which had previously been aged during OTR measurements and thus displayed very slow electrode kinetics. The results of OTR before and after treatment demonstrated an enhancement of 
electrode kinetics by over 10x after the treatment. However, the treatment works only for a $<24$ hours under testing conditions, after which TEM showed a dense La oxide layer $\sim 10 \mathrm{~nm}$ thick on the surface, as shown in figure 2. The improvement in electrode kinetics is believed to arise from a scavenging of impurities by La, thus opening free surface of PCO to reaction with oxygen. New methods to stabilize this improvement, as well as identifying the source of impurities, are underway.

\section{References}

[1] S. R. Bishop, J. Druce, J. J. Kim, J. Kilner, and H. L. Tuller, ECS Transactions, ECS Transactions, v. 50, no. 27, pgs. 35-38 (2013).

[2] S. R. Bishop, J. Druce, J. Kilner, T. Ishihara, and K. Sasaki, ECS Transactions, v. 57, no. 1, pgs. 2003-2007 (2013).

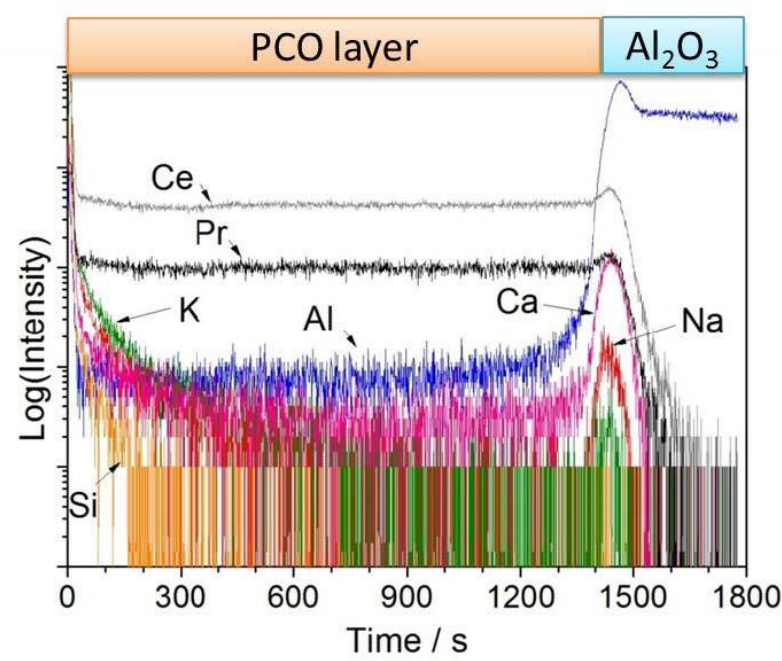

Figure 1: SIMS of post-OTR PCO film, from reference [1].

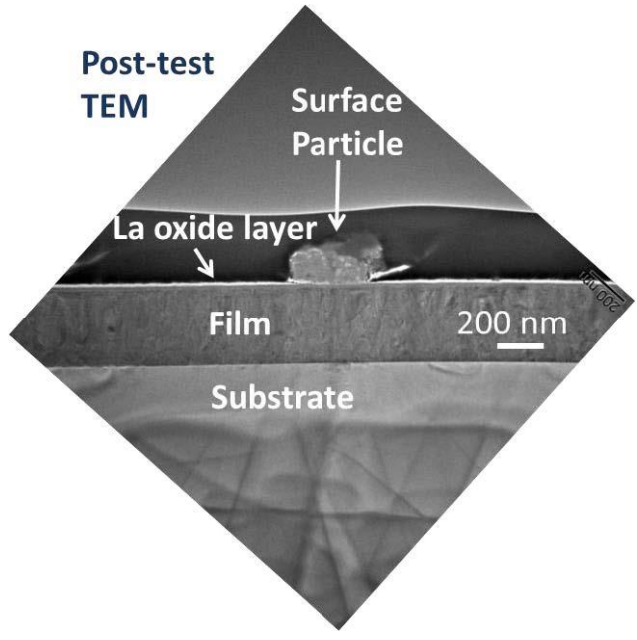

Figure 2: TEM cross-section of a post-OTR film with La oxide surface treatment. 